\title{
Antonio Pasquali y la Escuela de Frankfurt. Lecturas y apropiaciones (1966-1970)
}

\author{
Antonio Pasquali and the Frankfurt School. Readings and \\ appropriations (1966-1970) \\ Antonio Pasquali e a Escola de Frankfurt. Leituras e apropriações \\ (1966-1970) \\ Roberto Emiliano SANCHEZ NARVARTE, Argentina \\ Instituto de Investigación Aníbal Ford / emiliano.sanchez@perio.unlp.edu.ar \\ Serguei KOMISSAROV, Argentina \\ Universidad Nacional de La Plata / sergio.komissarov@perio.unlp.edu.ar
}

Chasqui. Revista Latinoamericana de Comunicación

N. ${ }^{o} 141$, agosto - noviembre 2019 (Sección Ensayo, pp. 257-274)

ISSN 1390-1079 / e-ISSN 1390-924X

Ecuador: CIESPAL

Recibido: 22-02-2019 / Aprobado: 16-11-2019 


\title{
Resumen
}

En este trabajo nos proponemos analizar las condiciones en las que Antonio Pasquali se aproximó, entre mediados de los años sesenta y principios del setenta, a las reflexiones filosóficas y sociológicas elaboradas por la Escuela de Frankfurt. Desde un marco teórico-metodológico inscrito en la sociología de la cultura, se reconstruyen las redes culturales e intelectuales en las que el teórico venezolano participó. Esta entrada analítica permite dar cuenta de la producción de ciertas lecturas y apropiaciones de la denominada "teoría crítica de la sociedad", en un contexto específico sobre las discusiones en torno a la relación entre la comunicación, la cultura y los medios masivos.

Palabras clave: Antonio Pasquali; Teoría de la Comunicación; Sociología de la cultura; Teoría crítica

\begin{abstract}
In this paper, we propose to analyze the conditions in which Antonio Pasquali approached, between the mid-sixties and the beginning of the seventies, the philosophical and sociological reflections elaborated by the Frankfurt School. From a theoretical-methodological framework inscribed in the sociology of culture, the cultural and intellectual networks in which the Venezuelan theorist participated are reconstructed. This analytical input allows us to account certain readings and appropriations of the so-called "critical theory of society", in a specific context about the discussions about the relationship between communication, culture and the mass media.
\end{abstract}

Keywords: Antonio Pasquali; Communication theory; Sociology of culture; Critical theory

\section{Resumo}

Neste artigo vamos a analisar as condições nas que Antonio Pasquali se aproximou, entre meados dos anos sessenta e princípios dos anos setenta, às reflexões filosóficas e sociológicas elaboradas pela Escola de Frankfurt. Desde uma abordagem teórica e metodológica situada na sociologia da cultura, são reconstruídas as redes culturais e intelectuais nas que o teórico venezuelano participou. Essa entrada analítica permite dar conta da produção de certas leituras e apropriações da denominada “teoria crítica da sociedade”, num marco especifico sobre as discussões em relação à comunicação, a cultura e os meios massivos.

Palavras-chave: Antonio Pasquali; Teoria da Comunicação; Sociologia da cultura; Teoria crítica 


\section{Introducción}

En este artículo, vamos a reconstruir las problemáticas intelectuales y culturales a partir de las cuales Antonio Pasquali se acercó a los trabajos de la Escuela de Frankfurt. Trazar el campo de dilemas desde el cual fueron leídos algunos textos de Horkheimer, Adorno y Marcuse, nos va a permitir, posteriormente, analizar qué apropiaciones realizó el teórico venezolano y de qué modo las incorporó a los debates locales.

Suele vincularse la figura de Pasquali a las ideas de Frankfurt desde la publicación de Comunicación y cultura de masas en 1964, (entre otros, Fuentes Navarro, 1991; Arroyo Gonçalves, 2005; Torres \& De los Reyes, 2009; Silva \& Campagnoli, 2010; Pineda de Alcázar, 2010 y 2014; Olmedo, 2011 y Martínez, 2016). De todos modos, y a contrapelo de las asociaciones teóricas habituales, Pasquali accedió fragmentariamente a trabajos de Marcuse recién en 1966 y esto operó como puerta de entrada a la lectura de Dialettica dell'illuminismo de Horkheimer y Adorno, en su versión italiana publicada por Giulio Einaudi Editore en ese mismo año (A. Pasquali. Entrevista personal Nº 1 . Febrero de 2015).

Para echar luz sobre este proceso, consideramos, se debe restituir la trama que operó como condición de posibilidad para que algunas reflexiones de la Escuela de Frankfurt llegaran a las manos de Pasquali. Para ello, vamos a indagar las redes y las formaciones culturales en las que el filósofo venezolano participó y que habilitaron la producción de ciertas lecturas e interpretaciones sobre la "teoría crítica".

\section{Marco teórico-metodológico}

Para la realización de este trabajo, retomamos las reflexiones que, desde la sociología de la cultura, han elaborado Roger Chartier y Raymond Williams. El historiador francés considera que, para el análisis de las "prácticas lectoras", la noción de "apropiación" acentúa la "invención creadora” en los procesos de recepción. Esto permite —en diálogo con Pierre Bourdieu (2012 [1979], p. 200)— pensar los "usos diferenciados" y los desplazamientos de sentido de las ideas, y enmarcar los empleos diversos de los bienes culturales en las disposiciones y en los hábitos de los itinerarios intelectuales de ciertos individuos o grupos culturales (Chartier, 1994, pp. 54-55).

Williams, por su parte, considera que las historias que sólo analizan los textos, construyen "versiones" de individuos que hicieron tal o cual obra que conllevan a un rápido etiquetamiento (Williams, 1997 [1989], p. 191). Para ello, propone desplazar la búsqueda de textos e individuos "desde arriba", es decir, partiendo de conceptualizaciones o posicionamientos intelectuales como un hecho naturalizado, hacia un análisis "de las relaciones específicas a través de las cuales las obras se hacen y se mueven” (p. 213). Esta entrada analítica inscribe la posición de los productores en procesos más amplios en los que las 
ideas emergen como "respuestas específicas" a los problemas sociales que los agentes están experimentando (p. 215). En este punto, Mariana Canavese (2015) propone que indagar el modo en que son "apropiadas" ciertas matrices teóricas, permite vincularlas a determinadas "experiencias concretas" y así reconstruir el "mapa de problemas" que hizo posible la producción de unas lecturas y no de otras (p. 30).

\section{La recepción de la "teoría crítica". Entre "Cine al día e Imagen"}

Hacia mediados de los sesenta, tras haber publicado Comunicación y cultura de masas (1964), Pasquali se situaba en una red heterogénea de formaciones y movimientos culturales. A través de su amigo, el crítico cinematográfico Alfredo Roffé, fue convocado a participar del primer Encuentro de Cine Nacional. Su investigación sobre el sistema de producción cultural y la postulación de la necesidad de formular políticas para la radio, la televisión y el cine, le otorgaban legitimidad para ingresar a ese grupo que se disponía a diagramar regulaciones para la actividad cinematográfica en Venezuela.

El movimiento que llevó adelante los encuentros de cine reunió a críticos/ as, cineastas, organismos culturales, universidades y productores/as con la finalidad de formular políticas para la actividad cinematográfica ${ }^{1}$. Los puntos de reunión fueron los Encuentros de Cine Nacional efectuados en 1966 en Ciudad Bolívar y en 1967 en Valencia y en Caracas. Allí, buscaban promover "una conciencia histórica y crítica acerca del cine como vehículo de cultura y de comunicación de masa" y la fundación de instituciones culturales que auspiciaran espacios para "las investigaciones históricas cinematográficas". Se asistía a un movimiento que no sólo legitimaba al cine como lenguaje "fundamental" del desarrollo cultural, sino que promovía la producción nacional en un contexto en el que los films que circulaban eran principalmente importados. En este sentido, el grupo de intelectuales criticaba el sometimiento del cine a la lógica comercial defendida por distribuidores y exhibidores que excluían e imposibilitaban el "advenimiento de una industria cinematográfica nacional" (Pasquali, 1972 [1967], p. 528). El resultado de los encuentros fue la formulación de un Proyecto de Ley de Cine redactado por Rodolfo Izaguirre, Alfredo Roffé, Sergio Facchi y Antonio Pasquali. El Proyecto establecía que "el cine, entre los medios colectivos de comunicación", era de "marcado interés social" y "ejercía influencia pública" (p. 528). De aquí que el Estado debía prestar apoyos económicos para el desarrollo de la industria cinematográfica nacional, favorecer su producción, distribución y exhibición.

1 Para profundizar en los alcances de estos procesos político-culturales, remitimos a los trabajos de Colmenares (1993 y 2014).

2 Pasquali incluyó las declaraciones y ponencias efectuadas en los encuentros, como anexo a Comunicación y cultura de masas a partir de su segunda edición en 1972 (pp. 597-611). 
La consolidación de este grupo de intelectuales suscitó, en 1967, la fundación de Cine al día $a^{3}$. Liderados por Roffé como director de la revista, el primer consejo de redacción estuvo conformado por Oswaldo Capriles, Sergio Facchi, Ambretta Marrosu y Antonio Pasquali, entre otros (s/f, 1967, p. 2). La participación de Pasquali en el proyecto remitía a su expertise académica: además de "teórico", había publicado distintos artículos en Cultura universitaria y había pertenecido al comité de redacción de Crítica contemporánea. Pasquali representaba una conexión con las problemáticas "culturales y sociológicas" acerca de la televisión, el contenido de los mensajes, la cuestión de la propiedad y el control de los medios (Colmenares, 1993, p. 146).

Desde Cine al día se afirmaba que si bien se había producido un proceso de "democratización de las obras" debido a los "avances tecnológicos en el campo de las comunicaciones", era importante pensar a los medios no solo como instrumentos de "difusión", sino como modos "aptos y propicios para la creación cultural" (s/f, 1967, p. 2). En este punto, el grupo se enfrentaba a una "dialéctica difícil": la de incorporarse a los procesos de producción cultural dominados por una "industria" que "homogeneizaba por vulgarización" los contenidos emitidos. La "industria cultural", se sostenía, era el "peor enemigo del hombre de cultura" (s/f, 1967, p. 2).

La revista transitó por distintas zonas de discusiones y habilitó a Pasquali a insertarse en redes de orden transnacional. Cine al día fue desplazando sus interrogantes sobre la "importancia del cine nacional en el proceso de desarrollo cultural" hacia una clave regional: la necesidad de fomentar, visibilizar y organizar el "nuevo cine latinoamericano" (s/f, 1969, p. 1) ${ }^{4}$. El dilema del pasaje del cine nacional como desarrollo cultural al tercer cine como militancia $y$ práctica política lo definía Oswaldo Capriles: el "tercer cine" buscaba "la revelación de la realidad", era el cine que en "formas diferentes combate por la liberación" contra una "dependencia" que "penetraba" todos los "campos de la vida latinoamericana" (1968p. 4).

En paralelo, Pasquali colaboró a lo largo de 1968 con la revista Imagen, a partir de su amistad con Guillermo Sucre, su director entre 1967 y 1968. Financiada por el Instituto Nacional de Cultura y Bellas Artes (INCIBA), la publicación tenía como objetivo dar a conocer al país las novedades sobre arte y literatura que se producían en el "mundo" (Sucre, 1968, p. 2)5.

Además de lo específicamente literario y artístico, Imagen incorporó como colaboradores académicos a Pasquali, Federico Riu y Eduardo Vásquez, para

3 Indicativo de que era una problemática en aumento al interior del campo intelectual venezolano, es que previo a Cine al día, entre 1964 y 1966 había salido en Caracas la revista Cine Teatro. Para profundizar en distintos aspectos de esta revista, ver Aguirre (2012, pp. 77-80).

4 Como sostiene María Luisa Ortega (2016), era un contexto de intensa actividad cinematográfica en la región, con la organización de filmotecas, cinematecas y encuentros.

5 Una de esas redes fue establecida con el grupo cultural organizado en torno a la revista argentina Sur, a partir de la amistad de Liscano y Sucre con Héctor Murena. De este modo, fue común el intercambio entre las novedades y traducciones argentinas y venezolanas en ambas revistas. 
escribir sobre novedades filosóficas. Las páginas principales en esta materia fueron dedicadas a Herbert Marcuse, quien había adquirido reconocimiento a partir de su participación en las protestas estudiantiles desarrolladas en la Universidad de Berkeley en $1964^{6}$. Unas conferencias que el filósofo alemán había dictado en febrero de 1966 en la Universidad Autónoma de México (UNAM) fueron, también, una de las vías por las cuales sus ideas llegaron a la revista ${ }^{7}$.

Uno de los primeros textos que Imagen le dedicó a Marcuse fue una reseña de Vásquez del libro Cultura y sociedad, que había sido editado por la editorial Sur en Buenos Aires en 1967. Vásquez señalaba que los artículos allí compilados eran centrales para comprender el lugar que debía ocupar la "teoría crítica" para pensar los procesos sociales. Si la "cultura afirmativa" —argumentaba Vásquez - tenía como "misión mantener el orden existente mediante una formación interior de los individuos", la "teoría crítica" tenía que estar "contra los hechos", "contra la cosificación" (Vásquez, 1967, pp. 6-7).

La revista se constituyó como un espacio de mediación entre Caracas y distintos puntos de la producción intelectual latinoamericana y europea. Posibilitó la circulación y el conocimiento de "nuevas" referencias en los planos artísticos, literarios y filosóficos, reseñando críticamente las nuevas publicaciones y constituyéndose en material de consulta para quienes quisieran "estar al día". Imagen promovió, además, una destacada labor de traducción. Contaba en su staff con la traductora y ensayista Julieta Fombona Zuloaga, quién se encargó de traducir textos, entre otros, de Lucien Goldmann y Raymond Aron. De hecho, a pedido del Instituto de Estudios Políticos de la Facultad de Derecho de la Universidad Central de Venezuela (UCV), tradujo en 1967 la primera edición al español de una de las obras clave de Marcuse, Razón y revolución. Hegel y el surgimiento de la teoría social.

La participación de Pasquali en ambos proyectos revisteriles le permitió conectarse con tendencias y debates de diversa índole, conocer experiencias político-culturales y a distintos realizadores y films. Con los intelectuales vinculadosal cine, en particular,incursionó enlasproblemáticas delaproducción cinematográfica latinoamericana. Su colaboración en Imagen lo conectó con la actualidad y los debates del pensamiento filosófico. Se entrecruzaban las discusiones sobre el rol del cine en la producción cultural, sobre los "efectos" de la cultura de masas y sobre la participación de la cinematografía en la formación de una "cultura nacional". Esta trama indica, en suma, que, para una franja de

6 Pasquali, Antonio. Entrevista personal N². Febrero de 2016. Caracas, Venezuela. Pasquali sostiene que tuvo un acceso fragmentario a sus obras, luego del "movimiento estudiantil de Berkeley, donde Marcuse fue una estrella". Después de leer algunos de sus textos, afirmó, "fui descubriendo a todos".

7 En 1966 la Escuela de Ciencias Políticas de la UNAM invitó a Erich Fromm a dictar una serie de conferencias. Junto a él participaron Irving Horowitz, André Gorz, Herbert Marcuse y Víctor Flores Olea. Las conferencias fueron compiladas en el libro E. Fromm y otros, La sociedad industrial contemporánea (Siglo XXI, 1967). 
la intelectualidad, la cultura era una preocupación política. En este marco, y como veremos más adelante, algunos trabajos de Frankfurt serían leídos en dos niveles: como un diagnóstico crítico del carácter industrializado de la cultura y, también, como una guía general para imaginar — vía la intervención políticaun horizonte cultural diferente.

\section{4. "El aparato singular" en las tramas de la renovación universitaria}

Pasquali escribió, en 1967, El aparato singular. Análisis de un día de TV en Caracas (UCV). Si bien afirmaba que era un trabajo que sólo "ponía al día" las estadísticas presentadas en Comunicación y cultura de masas (Pasquali, 1967, p. 19), allí se pueden leer una serie de desplazamientos relevantes en el posicionamiento teórico de su autor.

El trabajo fue publicado en un contexto en el que, en los pasillos de la UCV, se vivían intensamente las discusiones sobre comunicación y política. Elizabeth Safar —alumna en la Escuela de Periodismo a mediados de los sesenta-sostiene que había una estrecha relación entre la academia y el campo político. Quienes dirigían la Escuela de Periodismo en aquellos años "eran militantes: Héctor Mujica, Federico Álvarez y Luis Aníbal Gómez” ( E. Safar, Entrevista personal N ${ }^{\circ}$ 3. Marzo de 2016).

La universidad, además, estaba atravesada por un proceso de renovación que, entre otras cuestiones, discutía la relación entre el saber y la política. Este proceso no negaba la relación entre saber y política, sino el modo que había asumido dicha relación en la academia venezolana. La universidad tenía que replantearse sus condiciones de producción de conocimiento para que la ciencia ocupara un rol "revolucionario" destinado a las "necesidades del pueblo en su toma de conciencia” (Núñez Tenorio, 2009 [1968], p. 255). La discusión en torno a la necesidad de romper lazos de "dependencia" con los modos de producir y los marcos de interpretación norteamericanos configuraban toda una geopolítica del conocimiento que, a escala regional, implicaba la búsqueda de un reposicionamiento de las instituciones universitarias y de los agentes en ellas involucrados. Inscrito en estas discusiones, Pasquali planteaba la necesidad de romper los vínculos con el llamado "funcionalismo norteamericano" (Pasquali, 1967, p. 26).

Elaparatosingular esuntrabajoquepermitedarcuentadelreposicionamiento del autor en cuanto al marco de interpretación utilizado y del crecimiento de la "red nacional" de investigadores y grupos de trabajo sobre comunicación y medios. En una larga nota al pie, reconocía que el problema de los efectos en el plano "ideológico, motivacional y cultural" estaba siendo investigado en la Escuela de Psicología de la UCV y en la Escuela de Periodismo de la Universidad de Zulia. Si bien afirmaba que no se habían "practicado estudios sistemáticos de 
efectos", los primeros ensayos demostraban "resultados devastadores" (p. 99) ${ }^{8}$. Este proceso da cuenta de la configuración de redes de intercambio de ideas, de circulación de autores y obras y, por ende, de instancias de legitimación al interior del campo de estudios de comunicación en una trama de problemas y perspectivas de investigación que iba a ir conformando una tradición intelectual orientada por la pregunta sobre la relación entre cultura, medios y política.

En términos conceptuales, al iniciar El aparato singular con una larga cita de La ideología alemana de Karl Marx y Friedrich Engels, Pasquali comenzaba con el acto de "replantear" su "discurso" sobre el problema de la comunicación, los medios y la cultura de masas (p. 19). La entrada marxista le permitía situar a los medios masivos en un "problema cultural" más amplio: pensarlos como la "fábrica más eficiente de nuestras actitudes fundamentales ante la vida y los valores" (p. 26). Este desplazamiento era posibilitado por el encuentro con los trabajos de Horkheimer, Adorno y Marcuse, en tanto articulaban la cuestión de la ideología a la de la producción cultural masiva y la reproducción de las relaciones sociales. De todos modos, más que una incorporación rigurosa de los referentes de Frankfurt a su marco de interpretación - a los que había accedido recientemente - se lee un anudamiento en el cual la cultura y la comunicación no podían desligarse de las dimensiones económicas y de eso que habían dado en llamar "industria cultural" (p. 41).

La "presencia de Frankfurt" en El aparato singular se daba a partir de Dialettica dellilluminismo y de Razón y revolución. Pasquali incorporó fragmentos de dichos textos al inicio de cada capítulo. El segundo iniciaba con un pasaje en el que Adorno y Horkheimer afirmaban que, desde una perspectiva "técnica y cultural", la "publicidad y la industria cultural" eran equivalentes en tanto se desarrollaban con los mismos esquemas y procedimientos "para el manejo de los hombres" (p. 41). Esta entrada le permitía al teórico venezolano recuperar la noción de "dirigismo cultural" trabajada en Comunicación y cultura de masas, pero revisada y transformada: ahora se trataba de la producción de objetos culturales desde los criterios "del advertising-business, confeccionados con técnicas motivacionales" (p. 28, el destacado nos pertenece). El último capítulo comenzaba con un fragmento de Razón y revolución que postulaba el carácter estratégico del pensamiento dialéctico en la articulación entre práctica política y producción de conocimiento: como método que "debilitaba" la "siniestra confianza en el poder" y como arma que condujera al "derrumbe catastrófico del estado de cosas reinante" (p. 87).

Los acercamientos fragmentarios a la Escuela de Frankfurt habilitaron una ruptura con la investigación norteamericana. Si en Comunicación y cultura de masas el pensamiento estadounidense — de la mano de Lasswell- era

8 Además de reconocer el grupo de trabajo dirigido por Sergio Antillano y Martha Colomina en la Universidad de Zulia, la mayoría de las investigaciones que recuperó eran inéditas. Entre ellas, Margarita D’Amico, Los medios de comunicación en Venezuela (1964) y de Eduardo Santoro, La TV venezolana y la formación de estereotipos en el niño (1966). 
incorporado productivamente, a mediados de los años sesenta, al "pensamiento positivo" - a la luz de la crítica de Marcuse- debía oponerse un pensamiento dialéctico "que derribara la seguridad y la satisfacción del sentido común" (p. 87). Asumir esta perspectiva implicaba reposicionarse como investigador "ante el factum" de la "realidad comunicacional" (p. 119, -). De ahí, la creciente sospecha ante la descripción "objetiva" y no comprometida que proponían los "manuales de mass-communications". Pensar los medios, sostenía Pasquali, desde sus "funciones" a partir de su "significado empírico inmediato" era desconocer la realidad comunicacional venezolana, era no preguntarse qué significaba "aquí y ahora [la] televisión”. Su sentido básico, aseguraba en clave marxista, lo podía "revelar un análisis de su infraestructura económica" en tanto "fenómeno de naturaleza esencial y exclusivamente comercial" (p. 27,).

La relectura de Marx desde las perspectivas de Adorno, Horkheimer y Marcuse, le permitieron a Pasquali realizar varias operaciones: primero, en términos analíticos, pensar los medios masivos en el marcogeneral delaindustria cultural, como modo de producción sometido a los imperativos de la eficiencia y la técnica; segundo, inscribir los análisis de contenido de los medios en relación con las necesidades comerciales de las empresas; tercero, reposicionarse de un modo radical frente al "discurso científico" norteamericano. Pasquali sostenía que no "existían análisis sin juicios de valor", y se preguntaba cuánta "sociología norteamericana de las comunicaciones", tras pregonar "el cientificismo y la objetividad", se había convertido en "vademécum de demagogos y fabricantes de cuñas" (p. 29).

Estas primeras reelaboraciones a la luz de las lecturas frankfurtianas, más que una profunda revisión teórico-metodológica, eran apelaciones y "declaración de intenciones" de hacia dónde debía dirigirse la investigación en comunicación. Para el análisis de contenido, de hecho, Pasquali utilizaba la "descripción objetiva" de Bernard Berelson, dejando a un lado "el recurso a enfoques antropológicos, sociológicos y psicológicos” (p. 31). El modo en que operó el pasaje de la "adhesión simbólica" a una "incorporación efectiva" de la teoría crítica a su marco de interpretación es lo que vamos a trabajar en los apartados siguientes.

\section{Repensar la praxis. Desvíos y rupturas teóricas}

Ahora bien, ¿por qué Pasquali pudo mostrarse sensible a esta literatura? En principio, se podría explicar la recepción favorable de trabajos como Dialettica dell'illuminismo y Razón y revolución, sosteniendo que su formación filosófica produjo una predisposición y una inclinación —en el sentido de habitus - a un tipo de reflexión especulativa, característica de las producciones del campo filosófico, en un contexto de apertura hacia el análisis de múltiples problemáticas sociales y culturales. Al igual que Pasquali, los filósofos Federico Riu y Eduardo Vásquez compartieron ese acercamiento a las producciones frankfurtianas y 
también a las reflexiones - que si bien no pueden plantearse en términos de equivalencia o convergentes- de otra figura del marxismo heterodoxo como Georg Lukács y sus trabajos Historia y consciencia de clase (1923) y El asalto a la razón (1954) $)^{9}$. Esta "apertura" de la filosofía a pensar distintos aspectos de lo social lleva, en segundo lugar, a conjeturar que las problematizaciones de Pasquali se conectaron con las indagaciones de los filósofos de Frankfurt desde otra clave: la reconfiguración de los procesos de producción cultural en un momento en que el capitalismo se había convertido en "una gigantesca maquinaria monopólica" (Horkheimer \& Adorno, 2009 [1944], p.169).

La relación de Pasquali con las investigaciones del Instituto de Investigación Social de Frankfurt residiría, asimismo, en un plano político-ideológico; una relación que debe entenderse en el contexto de una problemática teóricopolítica relativa a la emergencia de la sociedad de masas como también al carácter dependiente de los medios de comunicación. Esta condición determinaba "ideológicamente" la producción de contenidos audiovisuales y su carácter "alienante". Con todo, y como veremos a continuación, la relación con Frankfurt se abrirá también en un plano teórico-metodológico desde el cual pensar los procesos de producción cultural.

Pasquali incorporó las reflexiones de Marcuse y Adorno en dos trabajos que produjo a lo largo de 1968: un breve artículo publicado en el $\mathrm{N}^{\circ} 29$ de la revista Imagen y en una ponencia que presentó en el XIV Congreso Internacional de Filosofía realizado en Viena. En Imagen, publicó el artículo "Por Marcuse y la utopía". Allí, afirmaba que El hombre unidimensional era "un intento riguroso por revivir la utopía política" y lo consideraba como "uno de los dos o tres escritos de filosofía más importantes del siglo". Sostenía que la obra de Marcuse debía ser leída desde "la dialéctica de la ideología y de la utopía" desarrollada por Karl Mannheim (Pasquali, 1968a, p. 7) ${ }^{10}$. La propuesta de Marcuse, en términos teóricos, era un recurso crítico frente a las posiciones "anti-posibilistas" que devenían en "uno de los residuos filosóficos de la unidimensionalidad ideológica". En términos políticos, significaba una reapertura "a lo posible contra el inmovilismo ideológico-naturalista": dinamismo dialéctico contra las "lógicas del dominio" (p. 8).

9 Trabajo al que Pasquali había acudido para la elaboración de su tesis doctoral, publicada en 1963 bajo el título Fundamentos gnoseológicos para una ciencia de la moral (EBUC, Caracas). Allí Pasquali recuperaba las reflexiones de Lukács para realizar una genealogía del irracionalismo como método cognoscitivo. Riu publicó, en 1968, Historia y totalidad (Caracas, Monte Ávila), un breve ensayo sobre el concepto de reificación en Lukács, en el que analizaba su potencialidad explicativa de los procesos sociales.

10 En Ideología y utopía (1966 [1929]), Mannheim había planteado que la "ideología" reflejaba hasta qué punto "el pensamiento de los grupos dirigentes" podía estar "tan profundamente ligado a una situación que, por sus mismos intereses", eran incapaces de visualizar ciertos hechos, volviéndose una "representación" que buscaba mantener "el existente orden de cosas" (p. 89 y p. 261). La "utopía", en cambio, trascendía la situación social y pretendía, mediante una "actividad de oposición, transformar la realidad histórica existente" (p. 265). 
Para la asistencia al Congreso de Filosofía en Viena, presentó una ponencia titulada "La philosophie pratique et la médiation de l' analyse sociologique" El positivismo, afirmaba allí, postulaba que la filosofía estaba perdiendo la capacidad de sintetizar el "mundo" como totalidad de los fenómenos y también "su función histórica de productora de ideologías destinadas a la justificación o a la transformación de la realidad". Esto era producto de la "subdivisión del trabajo intelectual y la especialización metodológica" que, en su conjunción, comprometían el "universalismo" de la filosofía "y su inclinación a la crítica de la cultura" (Pasquali, 1968b, p. 1). Ejemplo de ello, sostenía, era lo que ocurría con la denominada filosofía de la práctica, es decir, aquella que se preguntaba "por la relación entre [la] acción y [la] reflexión moral". El distanciamiento entre acción y reflexión filosófica, podía reconstituirse a partir de la incorporación al campo filosófico de la "mediación sociológica". Con esta idea, Pasquali hacía referencia a los estudios que analizaban el campo de la "interrelación humana", y situaba allí a los "análisis sociológicos definitivamente concluyentes de Mills, Horkheimer, Adorno y Marcuse" que debían "ser asumidos por la investigación" filosófica "porque eran los mejores frutos de esa mediación” (p. 2).

Si la filosofía estaba perdiendo su capacidad totalizadora y su función de crítica ideológica, antes que "renunciar" a ello, se tenían que reformular los "sistemas categoriales" y los "esquemas conceptuales para la comprensión de las nuevas formas de la praxis" (p. 3). Si se quería "dar razón del hombre histórico", se debían "tomar en consideración los mejores resultados teóricos y metodológicos conseguidos por las ciencias sociales" para "reactualizar los esquemas operativos" que habilitaran la comprensión ética de la praxis (p. 6).

Para construir el campo de problemas, Pasquali tomaba como punto de partida los planteos de Dialettica dell'illuminismo y de El hombre unidimensional. Recuperaba el "diagnóstico cultural" realizado por Horkheimer y Adorno en tanto permitía dar cuenta de la creciente incapacidad del sujeto de "pensar el pensamiento", de la "pérdida de conciencia" autorreflexiva en un contexto de "dirigismo social ejercido mediante el control de los canales de comunicación masiva" impuesto por el "iluminismo". Este — siguiendo a Marcuse- no asumía las formas del "despotismo armado", sino que se configuraba a través del condicionamiento llevado adelante por "la clase dominante" al rechazar aquellas ideas, aspiraciones y objetivos que trascendieran el universo constituido (p. 6, destacado en el original). Este "dramático" cuadro social llevaba a Pasquali a distanciarse -en consonancia con los frankfurtianos- del "entusiasmo de Marx por la aguda conciencia del proletariado" para romper con su "propia alienación" (p. 6). Situar el problema en estos términos le permitía afirmar que la "imposibilidad de dudar" en el sujeto contemporáneo era un "síntoma definitivo de la parálisis de la razón dialéctica". El "estado especial del hombre

11 Copia mimeografiada en español. La filosofía práctica y la mediación del análisis sociológico. Expediente "Pasquali Greco, Antonio Arnaldo", período "A". Archivo Histórico de la Facultad de Humanidades y Educación (UCV). Datos obtenidos el 19/02/2015, Caracas. 
alienado" lo cerraba a toda influencia crítico-negativa de lo existente volviéndolo "incomunicable". En este marco, el intelectual tenía que asumir la "urgente" tarea de emprender desde la filosofía de la práctica un replanteo de las categorías relacionales, revisar los modos habituales de referirse a los problemas sociales contemporáneos (p. 7).

El replanteo conceptual hacía referencia a la "tabla kantiana de las categorías" que el propio Pasquali había tomado para elaborar la analogía entre comunicación y comunidad desarrollada en Comunicación y cultura de masas. En este sentido, consideramos que cuando Pasquali, en su ponencia, planteaba si acaso la reflexión filosófica de Kant seguía siendo útil para la comprensión de la "reificación, [la] sociedad industrial avanzada, [la] planificación y [el] control", no estaba haciendo otra cosa que replantear sus propios esquemas de comprensión utilizados en Comunicación y cultura de masas. Las elaboraciones de Adorno, Horkheimer y Marcuse produjeron no sólo una desestabilización sino una ruptura con sus propias fundamentaciones teórico-filosóficas que habían servido en 1964 para producir "un sistema categorial de la relación" que pudiera explicar los procesos comunicacionales (Pasquali, 1964, p. 8). Como veremos en el pasaje siguiente, la incorporación de algunas ideas de estos filósofos alemanes en su marco de interpretación, constituyó además, la condición de posibilidad de una clave de lectura desde la cual analizar las novedades teóricas que llegaban al campo intelectual venezolano.

\section{Lectura de McLuhan desde el prisma frankfurtiano}

En la revista Imagen, se publicaron artículos y reseñas sobre Marcuse, y en menor medida sobre Louis Althusser, Jean-Paul Sartre y Roland Barthes. A las manos de la revista dirigida por Sucre, había llegado, también, la primera edición de Understanding Media, de Marshall McLuhan, que implicó una pronta lectura de Pasquali que fue sintetizada en una reseña publicada bajo el título "Marshall McLuhan o la ideología represiva" (Pasquali, 1968c).

El artículo se iniciaba con una reflexión sobre El hombre unidimensional. Si Marcuse, sostenía Pasquali, era representante de un "filón homogéneo de pensamiento negativo", el "pensamiento positivo" de McLuhan "era una franca ideología": sus ideas eran "representaciones falsas" y de "respaldo al orden social e intelectual predominante" (Pasquali, 1968c, p. 17). La lectura de McLuhan a contraluz de las reflexiones de Marcuse situaban la crítica de Pasquali en una dimensión teórico-política e inscribía a la producción del escritor canadiense como "breviario ideológico del pensamiento conservador" (p. 17). De aquí que el artículo pretendiera "desarmar algunas piezas fundamentales del aparato ideológico de McLuhan" y poner al descubierto las "representaciones" que se proponían "justificar el papel de la industria cultural en la civilización del bienestar" (p. 17). 
La crítica de Pasquali se situaba en los "aspectos centrales" del esquema teórico de McLuhan. Dichos aspectos eran la "desaparición" del concepto de "masa", la neutralización del concepto de "medium" y el "desinterés" por la pregunta acerca del "uso" de los medios. El concepto de "masa" era central para explicar los medios de comunicación: "era el componente sociológico que le otorgaba fuerza y sentido" y permitía pensarlos en su inscripción y rol en la sociedad. Quitarle la función masificante otorgada por Horkheimer y Adorno a la industria cultural, era una "hábil operación quirúrgica" que desplazaba su sentido crítico (p. 16, destacado en el original). Tras la lectura de Dialettica dell'illuminismo, Pasquali entendía que los medios masivos cumplían una tarea fundamental en la vida cotidiana como forma de distraer a los trabajadores en su tiempo libre. Pasquali planteaba que, al quitarle este espesor, McLuhan se inscribía en una trama de conceptualizaciones que sustituían a los medios masivos por nominaciones como "comunicación colectiva", "comunicación social" o "simplemente medios de comunicación", y devenía en un "esencialismo tecnológico" al que ya no podían "aplicarse esquemas éticos, políticos, sociológicos y económicos" (p. 16).

Esas "reformulaciones" conducían a Pasquali a entender que McLuhan proponía, con el concepto de "medium", una "neutralización" de los medios masivos. Con la acepción como "simple aparato o extensión de la subjetividad psicofísica" - seguía Pasquali- McLuhan pretendía "invalidar" los análisis de "fuentes, modos y efectos del mensaje" que se fundamentaban en la comprensión de la carga significante e intencional de los contenidos (p. 17). Los "significados de los contenidos", la "influencia" y el rol político de los medios, habían trazado el mapa de problemas configurado por esos años en la investigación en comunicación masiva en el campo académico e intelectual venezolano, y también en las problematizaciones que se llevaban adelante en los encuentros organizados por la CIESPAL ${ }^{12}$. Justamente, la pregunta por el uso de los medios, que según Pasquali se volvía "insignificante" en la obra de McLuhan, volvía inteligibles los procesos de masificación cultural orientados ideológica y políticamente por las clases dominantes (p. 17). El pensamiento de McLuhan en tanto "profeta del naturalismo", como "ideólogo de la conservación", finalizaba, podía llenar de "regocijo a la Asociación Interamericana de Radiodifusión y a los publicistas" porque era una teoría que justificaba ideológicamente las estrategias de los "gerenciales de la comunicación" (p. 18).

La lectura que Pasquali realizó de Understanding Media era sintomática de las condiciones de producción de conocimiento en comunicación y medios para una franja de la intelectualidad venezolana. Si el "problema político de los medios masivos" había ido in crescendo con el correr de los años sesenta, lo fue en equivalente proporción el posicionamiento crítico frente a la masificación de la cultura y los efectos por ella producida. Las investigaciones publicadas

12 Ver, por ejemplo, el documento editado por la CIESPAL, Utilización de los medios de Información en Quito (1966). 
entre 1964 y 1968 pretendían dar cuenta de cómo los medios influían política e ideológicamente en la sociedad. Esa continuidad en el modo de pensar la relación entre medios de comunicación, cultura y política, se vio "radicalizada" con la incorporación de algunas reflexiones de la Escuela de Frankfurt. En la lectura que Pasquali hizo de McLuhan, consideramos, se lee el modo en que esa deriva colectiva se inscribió en su propia formación e itinerario intelectual.

\section{La teoría crítica en clave "comunicacional"}

Las reconfiguraciones del campo académico e intelectual hacia finales de los sesenta, se produjeron en un marco de intensos movimientos culturales que tuvieron dos importantes polos de tracción. Por un lado, la consolidación de la industria editorial local con la fundación de la empresa estatal Monte Ávila en julio de 1968, creada por el INCIBA, cuyo primer director fue Benito Milla. En 1969, Monte Ávila publicó la compilación Industria cultural y sociedad de masas y de Adorno Intervenciones. Nueve modelos de crítica. En 1970, del mismo autor, publicó Sobre la metacrítica de la teoría del conocimiento ${ }^{13}$. Monte Ávila no sólo se revelaba como un dispositivo importante en la tarea de traducción y edición de obras extranjeras, sino también como institución clave para la visibilización y profesionalización de los escritores locales que podían firmar contratos con una empresa que tenía como horizonte el mercado cultural de la región.

Otro de los polos de tracción de este proceso de cambio fue el movimiento de renovación universitaria que, como dijimos, buscaba replantear la relación entre el saber y la política, y establecía una crítica en planos más amplios respecto a los marcos de comprensión de la realidad social, específicamente al funcionalismo y al empirismo norteamericano. En este punto, Negrón (2005) sostiene que entonces se produjo una apertura a diversas matrices que, si bien eran de corte mas o menos de izquierda, se presentaban como una crítica al llamado "marxismo tradicional-ortodoxo"(p. 88). La renovación generó transformaciones internas en toda la universidad.

En el caso de la Escuela de Periodismo de la UCV, se planteó, a la luz de las discusiones que se estaban dando, que se debía cambiar el nombre de la institución y denominarse Escuela de Comunicación Social (AAVV, 1987, pp. 91-93). Las autoridades consideraban que el nuevo plan trabajaba "distintas ramas de la Comunicación Social" que no eran "actividades específicamente periodísticas". La implementación del nuevo plan respondía a las necesidades

13 Estas condiciones específicas de finales de los sesenta configuraron un contexto importante de interpretaciones, traducciones y circulación de textos de la Escuela de Frankfurt. Uno de los polos clave se situó en Argentina y se expresó desde el proyecto cultural articulado en torno a la revista Los Libros. La revista, dirigida por Héctor Schmucler, publicó artículos y/o publicidad sobre obras de la Escuela de Frankfurt de forma ininterrumpida a lo largo de sus primeros 10 números entre julio de 1969 y agosto de 1970. Sobre la proliferación de publicaciones de distintos autores de la Escuela de Frankfurt en América Latina hacia los años sesenta, ver Entel, Gerzovich \& Lenarduzzi (2005 [1999]). 
de revisar la "función de la escuela" en relación con las "recomendaciones" de la CIESPAL en torno a la "diversidad de funciones del Periodismo" y a la "vinculación de la enseñanza con la actualidad económica, social y cultural". Este proceso que atravesó la UCV se dio de forma complementaria a otras escuelas, como la de Zulia, y se puede afirmar que fue un punto alto del proceso de institucionalización de las redes de investigación que venían tejiéndose desde mediados de los sesenta.

En ese marco y tras un acuerdo con la editorial Monte Ávila - a través de su amigo Juan Liscano-, se reeditó en 1972 Comunicación y cultura de masas, cuyo prefacio fue escrito por Pasquali en 1970. Allí planteó, en líneas generales, qué de la teoría crítica podía ser pensado en clave "comunicacional". Afirmaba que, entre la primera y la segunda edición de su trabajo, "demasiadas novedades teóricas, demasiados aportes capitales" habían "recibido la filosofía social y la sociología de las comunicaciones" como "para no sentir" "los límites de un escrito nacido" antes de esas contribuciones teóricas (Pasquali, 1972 [1970], p. 13). De hecho, sostenía Pasquali, acceder a ciertos trabajos en 1964 le "hubieran impuesto un más complejo y articulado planteamiento del problema”. Por eso —y si bien no lo hizo — la "parte teórica" del trabajo merecía ser "desmantelada" (p. 13).

Para pensar el "proceso de masificación" se volvían necesarias las reflexiones de Frankfurt, aun cuando salvo en "textos menores", no le habían "concedido una real prioridad" a las comunicaciones masivas. Al respecto, sostenía, quien los "haya leído en clave de 'comunicación", habría constatado el "renovado trasfondo conceptual que ofrece la teoría crítica a un análisis de la información social". Fundamentalmente, el concepto de "industria cultural" permitía analizar cómo las grandes empresas "intervenían la cultura" para mantener las relaciones sociales y perpetuar "los principios de realidad". En este marco, "los medios masivos" eran "la punta de lanza de una tecnología" que era la "expresión suprema de la razón instrumental y represiva" (p. 29).

\section{Conclusiones}

Trazar el mapa de dilemas que atravesó a una franja de la intelectualidad venezolana interpelada por los problemas vinculados a los medios masivos y a la producción cultural, permite dar cuenta de las condiciones a partir de las cuales algunas ideas — no sólo de la Escuela de Frankfurt - fueron operativas para la crítica cultural y para imaginar otras relaciones entre la producción de conocimiento y la práctica política. Por otro lado, permite dar cuenta de la constitución de una serie de vínculos institucionales trasnacionales que facilitaron el movimiento de ideas en torno a la relación comunicación, política y cultura.

Quisiéramos indicar, al menos, que la apropiación de algunos trabajos de la Escuela de Frankfurt se dio en el marco, además, de los primeros intentos 
de organizar encuentros de investigadores de la comunicación, cristalizados finalmente en Maracaibo en junio de 1970. En el Primer Encuentro de Investigadores de la Comunicación Colectiva, se planteó la necesidad de difundir estudios y la prioridad de establecer agendas de investigación. En este sentido, afirma Aguirre (1996), las preguntas se desplazaron desde la crítica a las funciones que cumplían los medios de comunicación de masas hacia las condiciones que posibilitaban su contribución al desarrollo social y a la emancipación política. El cruce entre crítica teórica y praxis política se formulaba en consonancia con unas tramas regionales en las que los estudios sobre comunicación, medios y cultura, se inscribían en un amplio "movimiento crítico y revitalizador de las ciencias sociales" (Sánchez Ruiz, 1992, p. 19).

La obra de Pasquali comenzó a circular por fuera de Venezuela a partir de que sus trabajos fueran editados por Monte Ávila. Tanto la segunda como la tercera edición de Comunicación y cultura de masas (1972 y 1976), habilitaron a que su figura se asociara con las reflexiones frankfurtianas, en un contexto de emergencia de la comunicación y los medios como problema teórico y político.

El proceso universitario de formación de redes con distintas instituciones se dio de manera paralela a la incorporación de Pasquali al plantel de escritores de Monte Ávila que, con una estructura de circulación regional, fue estableciendo acuerdos de publicidad con distintas organizaciones y formaciones culturales, como el grupo de la revista Los Libros. Si bien no podemos profundizar sobre este aspecto, la circulación de algunas obras de Pasquali se produjo a partir de distintas redes que operaron como estructuras relacionales de intercambio de ideas. Podríamos decir que se configuró una constelación urbana con nodos en Ciudad de México, Caracas, Quito, Santiago, Buenos Aires y São Paulo que, en su trazado, fue conformando el territorio material de circulación de autores e ideas. La emergencia de espacios dedicados a la investigación vinculados a la comunicación, la cultura y la política —en el marco de específicas coyunturas nacionales-, la consolidación de redes editoriales que habilitaron una importante fluidez en la circulación de libros, y el establecimiento de convenios de intercambio entre formaciones culturales —entre Imagen y Sur, o Los Libros con Monte Ávila y EBUC, por ejemplo- generaron las condiciones propicias para que la obra y figura del filósofo venezolano comenzara un proceso de conocimiento y legitimación a escala transnacional.

\section{Referencias bibliográficas}

AAVV. (1987). Materiales para la historia de la Escuela de Comunicación Social. Caracas: UCV. Aguirre, J. M. (1996). De la práctica periodística a la investigación comunicacional. Caracas: Universidad Católica Andrés Bello.

(2012). Estilos de la crítica cinematográfica: el cine para leer y su crisis. Comunicación. (157), 77-8o. Recuperado de http://gumilla.org/biblioteca/bases/biblo/texto/ COM2012157.pdf. 
Arroyo Gonçalves, C. (2005). Escuela latinoamericana de comunicación y el pensamiento crítico de Antonio Pasquali. ALAIC. Revista Latinoamericana de Ciencias de la Comunicación, 2(2), 22-30. Recuperado de https://www.alaic.org/revista/index.php/alaic/ article/view/129.

Bourdieu, P. (2012). [1979]. La distinción. Criterio y bases sociales del gusto. Argentina: Taurus. Canavese, M. (2015). Los usos de Foucault en la Argentina. Buenos Aires: siglo veintiuno.

Capriles, O. (1968). Mérida: realidad, forma y comunicación. Cine al día. (6) 4-9.

Chartier, R. (1994). Libros, lecturas y lectores en la Edad Moderna. Madrid: Alianza Universidad.

Colmenares, M. G. (1993). Contextualización de Cine al Día (1967-1983) y sus planteamientos en torno al cine venezolano y latinoamericano (Tesis de Grado). Copia mimeografiada.

Colmenares, M. G. (2014). La incorporación del cine a las políticas culturales del Estado (Venezuela, 1958-1982). 1 (26), 259-277.

Entel, A., Gerzovich, D., \& Lenarduzzi, V. (2005). [1999]. Escuela de Frankfurt. Razón, arte y libertad. Buenos Aires: Eudeba.

Fuentes Navarro, R. (1991). Un campo cargado de futuro. El estudio de la comunicación en América Latina. Guadalajara: ITESO.

Horkheimer, M., \& Adorno, T. (2009). [1944]. Dialéctica de la ilustración. Madrid: Taurus.

Mannheim, K. (1966). [1929]. Ideología y utopía. Madrid: Aguilar.

Martínez, R. (2016). Antonio Pasquali. Hacia una nueva comprensión comunicativa. ALAIC. Revista Latinoamericana de Ciencias de la Comunicación, 23 (12), 52-61. Recuperado de https://www.alaic.org/revista/index.php/alaic/article/view/74,4/391.

Negrón, J. (2005). Saber y poder. El proceso de renovación académica en la Escuela de Sociología y Antropología de la UCV (1967-1970) (Tesis de grado). Copia mimeografiada.

Núñez Tenorio, J. (2009). [1968]. Necesidad de una universidad nueva en Venezuela. En Renovar la renovación. Hacia la constituyente universitaria. 236-289. Venezuela: El perro y la rana.

Olmedo, S. (2011). Comprender la comunicación, de Antonio Pasquali. Razón y Palabra, (75), 1-31.Recuperado de http://www.razonypalabra.org.mx/N/N75/monotematico $75 / 27$ Olmedo M75.pdf.

Ortega, M. L. (2016). Las disyuntivas del documental. Las rupturas del 68 en el cine de América Latina, Mérida (68), 355-394. Argentina: Akal.

Pasquali, A. (1963). Fundamentos gnoseológicos para una ciencia de la moral. Caracas: EBUC. . (1964). Comunicación y cultura de masas. Caracas: EBUC.

. (1967). El aparato singular: análisis de un día de TV en Caracas. Caracas: UCV. . (1968a). Por Marcuse y por la utopía. Imagen, (29), 6-7.

. (1968b). Marshall McLuhan o la ideología represiva. Imagen, (27), 16-18. . (1968c). La filosofía práctica y la mediación del análisis sociológico. XIV Congreso Internacional de Filosofía, Viena. Copia mimeografiada. . (1972). [1964]. Comunicación y cultura de masas. Venezuela: Monte Ávila.

Pineda de Alcázar, M. (2010). Antonio Pasquali: la vigencia de su pensamiento cuarenta años después. Chasqui, 109, 18-20. Quito: CIESPAL. Recuperado de https://revistachasqui. org/index.php/chasqui/article/view/112

. (2014). Antonio Pasquali: la vigencia de su pensamiento cincuenta años después. Travesía intelectual de Antonio Pasquali. A propósito de los 50 años de Comunicación y cultura de masas, 21-30. Venezuela: UCAB.

Riu, F. (1968). Historia y totalidad. Venezuela: Monte Ávila. 
Sánchez Ruiz, E. (1992). Medios de difusión y sociedad. Guadalajara: Universidad de Guadalajara.

Silva, A. P., \& Campagnoli, M. A. O. Protagonismo de Antonio Pasquali na pesquisa-denúncia e sua influencia sobre a Escola Latino-americana de Comunicaçao. Chasqui, 109, 21-24. Recuperado de https://revistachasqui.org/index.php/chasqui/article/view/113.

s/a (1967). Cine y cultura en Venezuela. Cine al día, 1, 1-2.

s/a (1969). Cine del tercer mundo. Cine al día, 8, 3 .

Sucre, G. (1968). Primer aniversario. Imagen, 24, 2-3.

Torres, F., y De los Reyes, D. (2009). Rompecabezas de una obra: Antonio Pasquali y su utopía comunicacional. Caracas: UCAB.

Vásquez, E. (1968). 4 ensayos de Herbert Marcuse. Imagen, 6, 6-7.

Williams, R. (1997). [1989]. La política del modernismo. Buenos Aires: Manantial. 